Document downloaded from:

http://hdl.handle.net/10251/77009

This paper must be cited as:

Riedemann, J.; Peña Guiñez, R.; Cárdenas Dobson, R.; Blasco Giménez, RM.; Clare, J. (2014). Indirect matrix converter modulation strategies for open-end winding induction machine. IEEE Latin America Transactions. 12(3):395-401. doi:10.1109/TLA.2014.6827864

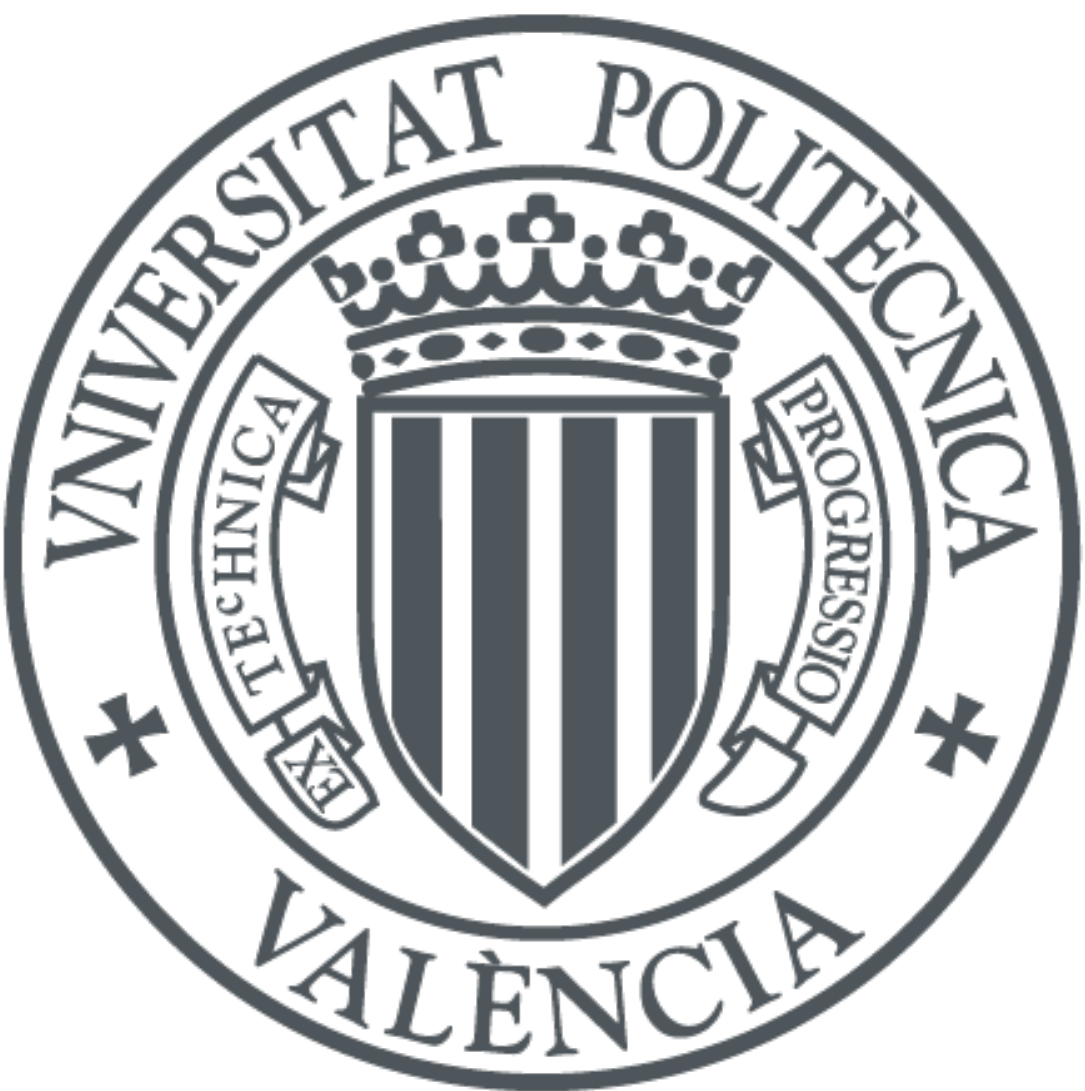

The final publication is available at

http://dx.doi.org/10.1109/TLA.2014.6827864

Copyright Institute of Electrical and Electronics Engineers (IEEE)

Additional Information 


\title{
Indirect Matrix Converter Modulation Strategies for Open-end Winding Induction Machine
}

\author{
J. Riedemann, R. Peña, Member, IEEE, R. Cárdenas, Senior Member, IEEE, R. Blasco-Gimenez, \\ Senior Member, IEEE, J. Clare, Senior Member, IEEE
}

\begin{abstract}
In this paper an Indirect Matrix Converter (IMC) topology suitable for controlling an open-end winding induction machine is presented. The IMC is a direct power converter having no bulky energy storage elements with an input or current rectifying stage and a voltage source inverter as an output stage. To provide energy to an open-end winding induction machine two output stages are needed. Then, it is possible to obtain a voltage across any phase of the load up to 1.5 times the input phase voltage without overmodulation. Two pulse width modulation strategies are presented: the first one is based on space vector modulation that suppresses the zero sequence voltage in the load whereas the second one is a carrier-based modulation which generates lower distorted input converter currents. Results are presented and discussed.
\end{abstract}

Keywords - open-end winding induction motor drive, matrix converter, zero sequence voltage.

\section{INTRODUCCIÓN}

$\mathrm{E}^{\mathrm{N}}$ $\mathrm{N}$ LOS últimos años, los esquemas basados en dos inversores de dos niveles (inversor dual) para alimentar motores de inducción de terminales abiertos han ganado interés debido a ciertas ventajas que poseen en comparación con inversores convencionales de tres niveles (NPC), tales como la ausencia de fluctuaciones del punto neutro y de diodos de enclavamiento. Sin embargo, el inversor dual puede causar la circulación de corriente de secuencia cero en la máquina [1] debido a la tensión de secuencia cero en las fases de la carga. Esta corriente de secuencia cero puede ser de gran amplitud si la impedancia de la carga es baja. Pueden utilizarse diferentes métodos para resolver este problema, como son la utilización de hardware adicional o estrategias especiales de modulación [2].

Los convertidores de enlace directo, también conocidos como Convertidores Matriciales, tienen la ventaja de no contar con elementos voluminosos para almacenar energía, pueden manejar flujos bidireccionales de potencia y, cuando alimentan máquinas de terminales abiertos, pueden conseguir hasta 1,5 veces la tensión de fase de entrada en las fases de la máquina [2].

J. Riedemann, Universidad del Bío-Bío, Concepción, Chile, jriedema@ubiobio.cl

R. Peña, Universidad de Concepción, Concepción, Chile, rupena@udec.cl

R. Cárdenas, Universidad de Chile, Santiago, Chile, rcd@ieee.org

R. Blasco-Gimenez, Universidad Politécnica de Valencia, Valencia España, r.blasco@ieee.org

J. Clare, University of Nottingham, Nottingham, U.K, jon.clare@nottingham.ac.uk
El Convertidor Matricial Indirecto (IMC) [3], consistente en etapas independientes de entrada y de salida, tiene ventajas similares al Convertidor Matricial Directo (MC). Este trabajo presenta una topología basada en un IMC para suministrar energía a una máquina de inducción de terminales abiertos. La topología utiliza dos etapas de salida como se muestra en Fig. 1 [4]. En este artículo, se presentan dos estrategias distintas de modulación: Modulación por Vectores Espaciales (SVM) y Modulación Basada en Portadora (SPWM). La estrategia SVM busca eliminar la tensión de secuencia cero en los terminales de la carga mientras la estrategia SPWM produce tensión de secuencia cero pero que solo contiene componentes de alta frecuencia. El modelo del convertidor de potencia y las estrategias de modulación son implementados usando cosimulación Matlab/Simulink-PSim.

\section{CONVERTIDOR MATRICIAL INDIRECTO CON DOS ETAPAS DE SALIDA}

La configuración del circuito propuesto se muestra en la Fig. 1. La etapa de entrada es un rectificador activo, consistente en seis interruptores bidireccionales [4,5], y que se controla para obtener una tensión máxima (positiva) en el enlace DC y un factor de desplazamiento unitario en la entrada. Dos inversores estándar (fuente de tensión) se usan como etapas de salida del IMC. Cada inversor puede producir ocho vectores espaciales, resultando en un total de 64 combinaciones de vectores espaciales para la topología dual.

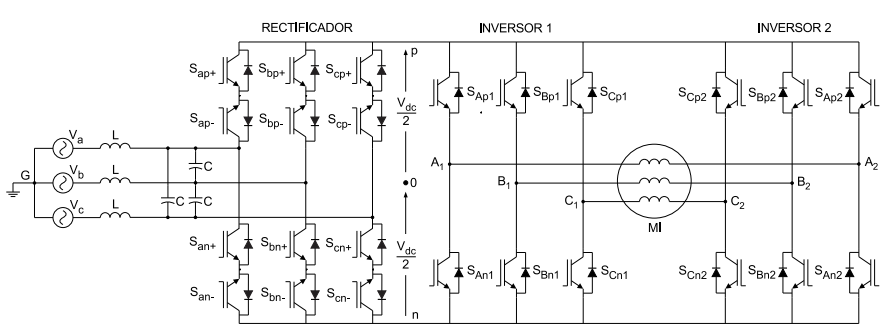

Figura 1. IMC de dos salidas alimentando un motor de terminales abiertos.

\section{MOdULACIÓN PARA LA ETAPA DE ENTRADA DEL IMC}

Para el rectificador de entrada del convertidor se utiliza modulación por vectores espaciales. Los ciclos de trabajo $\gamma-$ $\delta$ para esta etapa están dados por [4,5]:

$$
d_{\gamma}^{R}=\frac{d_{\gamma}}{d_{\gamma}+d_{\delta}} \quad, \quad d_{\delta}^{R}=\frac{d_{\delta}}{d_{\gamma}+d_{\delta}}
$$

donde

$$
d_{\gamma}=\sin \left(\pi / 3-\theta_{r e f, i}\right) \quad, \quad d_{\delta}=\sin \left(\theta_{r e f, i}\right)
$$


y $\theta_{\text {ref }, i}$ es el ángulo del vector de referencia.

\section{MODUlación PARA LAS ETAPAS DE SALIDA DEL IMC}

$\mathrm{El}$ inversor dual puede producir 64 vectores de tensión. Sin embargo, si se utilizan solo ciertas combinaciones de vectores del esquema dual, la tensión de secuencia cero en los terminales de la máquina puede eliminarse [6]. Si $V_{1}^{1}-V_{8}^{1}$ denotan los estados asumidos por el Inversor 1 y $V_{1}^{2}-V_{8}^{2}$ aquellos del Inversor 2, los vectores espaciales de los inversores individuales pueden escribirse como en la Tabla I.

TABLA I. ESTADOS DE CONMUTACIÓN DE LOS INVERSORES.

\begin{tabular}{|c|c|}
\hline $\begin{array}{c}\text { Estados Inversor } 1 \\
{\left[S_{A p 1} S_{B p 1} S_{C p 1}\right]}\end{array}$ & $\begin{array}{c}\text { Estados Inversor } 2 \\
{\left[S_{A p 2} S_{B p 2} S_{C p 2}\right]}\end{array}$ \\
\hline$V_{1}^{1}\left[\begin{array}{lll}1 & 0 & 0\end{array}\right]$ & $V_{1}^{2}\left[\begin{array}{lll}1 & 0 & 0\end{array}\right]$ \\
\hline$V_{2}^{1}\left[\begin{array}{lll}1 & 1 & 0\end{array}\right]$ & $V_{2}^{2}\left[\begin{array}{lll}1 & 1 & 0\end{array}\right]$ \\
\hline$V_{3}^{1}\left[\begin{array}{lll}0 & 1 & 0\end{array}\right]$ & $V_{3}^{2}\left[\begin{array}{lll}0 & 1 & 0\end{array}\right]$ \\
\hline$V_{4}^{1}\left[\begin{array}{lll}0 & 1 & 1\end{array}\right]$ & $V_{4}^{2}\left[\begin{array}{lll}0 & 1 & 1\end{array}\right]$ \\
\hline$V_{5}^{1}\left[\begin{array}{lll}0 & 0 & 1\end{array}\right]$ & $V_{5}^{2}\left[\begin{array}{lll}0 & 0 & 1\end{array}\right]$ \\
\hline$V_{6}^{1}\left[\begin{array}{lll}1 & 0 & 1\end{array}\right]$ & $V_{6}^{2}\left[\begin{array}{lll}1 & 0 & 1\end{array}\right]$ \\
\hline$V_{7}^{1}\left[\begin{array}{lll}1 & 1 & 1\end{array}\right]$ & $V_{7}^{2}\left[\begin{array}{lll}1 & 1 & 1\end{array}\right]$ \\
\hline$V_{8}^{1}\left[\begin{array}{lll}0 & 0 & 0\end{array}\right]$ & $V_{8}^{2}\left[\begin{array}{lll}0 & 0 & 0\end{array}\right]$ \\
\hline
\end{tabular}

Sea $V_{i j}=\left[V_{i}^{1} V_{j}^{2}\right]$ con $i, j=1 \ldots 8$, una combinación de vectores espaciales del inversor dual. La tensión de secuencia cero generada en los terminales de la máquina puede definirse como:

$$
v_{z S}=\frac{v_{A 1 A 2}+v_{B 1 B 2}+v_{C 1 C 2}}{3}
$$

En consecuencia, los vectores que no generan tensión de secuencia cero son [6]:

$$
\left[\begin{array}{l}
S_{A p 1} \\
S_{B p 1} \\
S_{C p 1} \\
S_{A p 2} \\
S_{B p 2} \\
S_{C p 2}
\end{array}\right]^{T} \in\left\{\begin{array}{lllll}
V_{88} & V_{55} & V_{53} & V_{35} & V_{33} \\
V_{44} & V_{51} & V_{31} & V_{46} & V_{42} \\
V_{15} & V_{13} & V_{64} & V_{24} & V_{11} \\
V_{66} & V_{62} & V_{26} & V_{22} & V_{77}
\end{array}\right\}
$$

Por lo tanto, para eliminar $v_{z s}$, la estrategia de modulación de los inversores puede emplear cualquiera de dos conjuntos diferentes, pero equivalentes en todo aspecto, de vectores espaciales activos:

$$
\begin{aligned}
& W_{1}=\left\{\begin{array}{llllll}
V_{15} & V_{35} & V_{31} & V_{51} & V_{53} & V_{13}
\end{array}\right\} \\
& W_{2}=\left\{\begin{array}{lllll}
V_{24} & V_{26} V_{46} & V_{42} & V_{62} & V_{64}
\end{array}\right\}
\end{aligned}
$$

\section{A. Modulación por Vectores Espaciales (SVM)}

En esta estrategia, la modulación se realiza usando los vectores activos del conjunto $W_{1}$ como vectores básicos. Estos vectores describen un hexágono dividido en seis sectores como muestra la Fig. 2b. Los vectores nulos se mapean dependiendo de la información del sector [2]. Este mapeo se muestra en la Tabla II.
TABLA II. MAPEO DE VECTORES NULOS.

\begin{tabular}{|l|c|c|c|c|c|c|}
\hline Sector & I & II & III & IV & V & VI \\
\hline Vector nulo & $V_{55}$ & $V_{33}$ & $V_{11}$ & $V_{55}$ & $V_{33}$ & $V_{11}$ \\
\hline
\end{tabular}

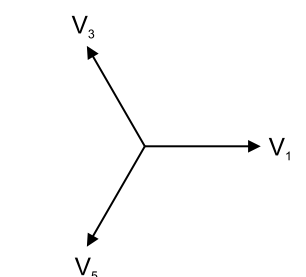

Vectores de tensión VSI 1

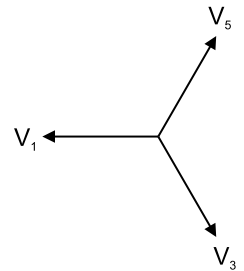

Vectores de tensión VSI 2

(a)

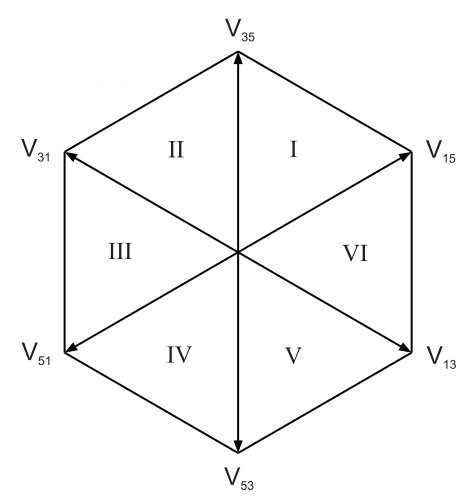

(b)
Figura 2. a) Vectores espaciales de los inversores individuales b) Combinaciones utilizadas de vectores espaciales.

Los ciclos de trabajo para la etapa de salida se calculan como:

$$
\begin{aligned}
& d_{\alpha}=m_{o}\left(d_{\gamma}+d_{\delta}\right) \sin \left(\pi / 3-\theta_{\text {ref }, o}\right) \\
& d_{\beta}=m_{o}\left(d_{\gamma}+d_{\delta}\right) \sin \left(\theta_{\text {ref }, o}\right) \\
& d_{0}=1-d_{\alpha}-d_{\beta}
\end{aligned}
$$

donde $\left(d_{\gamma}+d_{\delta}\right)$ se incluye para compensar las variaciones de la tensión en el enlace DC, $m_{o}$ es el índice de modulación $(0 \leq$ $\left.m_{o} \leq 1\right)$ y $\theta_{\text {ref,o }}$ es el ángulo del vector de referencia de tensión en la salida. Para obtener un correcto balance de las corrientes de entrada y las tensiones de salida en un período de conmutación, el patrón de modulación debe producir todas las combinaciones de estados de conmutación entre las etapas de entrada y salida [4], resultando en los siguientes ciclos de trabajo para los vectores activos:

$$
\begin{array}{lll}
d_{\alpha \gamma}=d_{\alpha} d_{\gamma}^{R} & , & d_{\beta \gamma}=d_{\beta} d_{\gamma}^{R} \\
d_{\alpha \delta}=d_{\alpha} d_{\delta}^{R} & , & d_{\beta \delta}=d_{\beta} d_{\delta}^{R}
\end{array}
$$

El ciclo de trabajo total de los vectores nulos es:

$$
d_{0, t o t}=1-d_{\alpha \gamma}+d_{\beta \gamma}-d_{\alpha \delta}-d_{\beta \delta}
$$

y los ciclos de trabajo combinados para los vectores nulos son:

$$
d_{0 \gamma}=d_{0, t o t} d_{\gamma}^{R} \quad, \quad d_{0 \delta}=d_{0, t o t} d_{\delta}^{R}
$$

La secuencia de conmutación se muestra en Fig. 3, conmutando la etapa de entrada con cero corriente DC. 


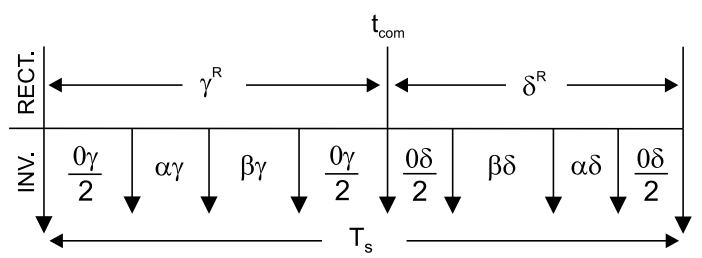

Figura 3. Secuencia de conmutación estándar para IMCs.

\section{B. Modulación basada en portadora}

En una estrategia PWM basada en portadora triangular (SPWM), los ciclos de trabajo para cada pierna del inversor 1 son:

$$
\begin{aligned}
& d_{a}=\frac{1}{2}\left(m_{o}\left(d_{\gamma}+d_{\delta}\right) \cos \left(\frac{2 \pi}{m_{f}} k\right)+1\right) \\
& d_{b}=\frac{1}{2}\left(m_{o}\left(d_{\gamma}+d_{\delta}\right) \cos \left(\frac{2 \pi}{m_{f}} k-\frac{2 \pi}{3}\right)+1\right) \\
& d_{c}=\frac{1}{2}\left(m_{o}\left(d_{\gamma}+d_{\delta}\right) \cos \left(\frac{2 \pi}{m_{f}} k+\frac{2 \pi}{3}\right)+1\right)
\end{aligned}
$$

donde $\left(d_{\gamma}+d_{\delta}\right)$ se incluye para compensar las variaciones de la tensión en el enlace DC, $m_{f}=f_{s} / f_{o}$ es el índice de frecuencia ( $f_{o}$ : frecuencia de salida, $f_{s}$ : frecuencia de conmutación), $m_{o}$ es el índice de modulación $\left(0 \leq m_{o} \leq 1\right)$ y $0 \leq k \leq m_{f}$. Los ciclos de trabajo del inversor 2 se obtienen usando (12)-(14) pero considerando un desfase de $180^{\circ}$ en las funciones coseno. Para propósitos de implementación, los ciclos de trabajo $d_{a}, d_{b}$ y $d_{c}$ son transformados en ciclos de trabajo equivalentes asociados a vectores de conmutación. Así, considerando la Fig. 4, los ciclos de trabajo $\alpha-\beta-0$ son:

$$
\begin{aligned}
& d_{0}=1-d_{\max } \quad, \quad d_{\alpha}=d_{\text {max }}-d_{\text {mid }} \\
& d_{\beta}=d_{\text {mid }}-d_{\text {min }} \quad, \quad d_{7}=d_{\text {min }}
\end{aligned}
$$

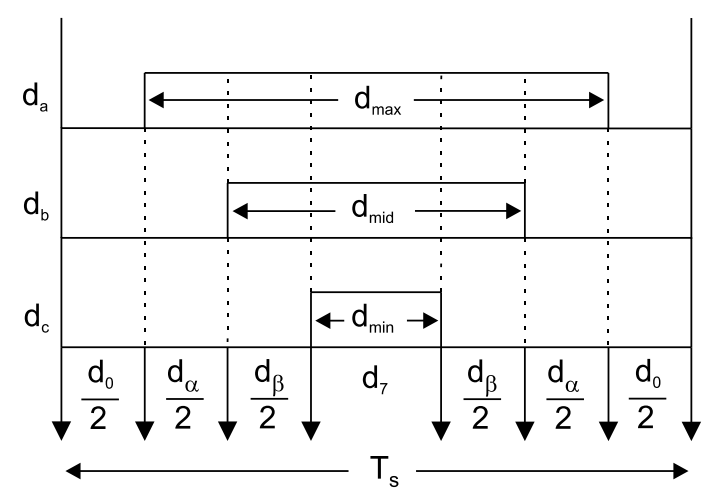

Figura 4. Ciclos de trabajo para un inversor individual.

Como se mencionó para la estrategia SVM, es necesario combinar los ciclos de trabajo del rectificador e inversores usando (9)-(11). En este caso, el ciclo de trabajo correspondiente al estado de conmutación $\quad\left[S_{A p} S_{B p} S_{C p}\right]=\left[\begin{array}{lll}0 & 0 & 0\end{array}\right]$ es:

$$
d_{00}=d_{0, t o t}-d_{\min }
$$

y finalmente, los ciclos de trabajo combinados para los vectores nulos son:

$$
d_{0 \gamma}=d_{00} d_{\gamma}^{R} \quad, \quad d_{0 \delta}=d_{00} d_{\delta}^{R}
$$

Los ciclos de trabajo de las etapas de salida se representan en la Fig. 5.

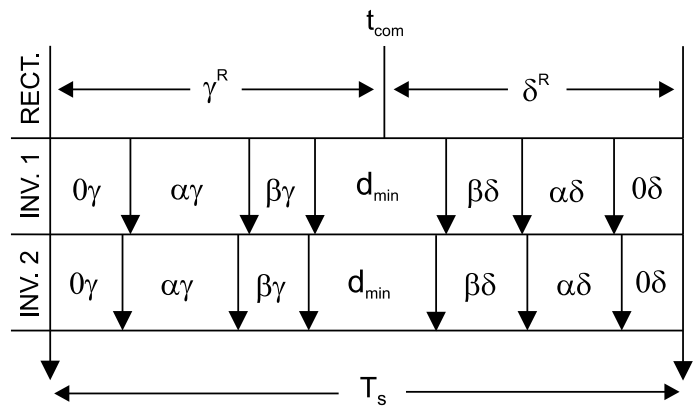

Figura 5. Ciclos de trabajo para los inversores.

\section{Plataforma de SimUlación}

El sistema se ha modelado usando una plataforma de cosimulación que comprende los software Matlab/Simulink y PSim. Usando este tipo de plataforma, se aprovechan las capacidades de Matlab/Simulink en la ejecución de algoritmos de modulación y manejo de señales de control. Por otro lado, el modelado del convertidor usando PSim es sencillo y las características de este software para simular circuitos electrónicos de potencia se aprovechan en su totalidad. Con motivo de sincronizar las plataformas de simulación Matlab/Simulink y PSim, y obtener resultados adecuados, es necesario utilizar los mismos (o al menos muy similares) tiempos de paso de simulación en ambos software. Para realizar la simulación, el modelo se separa en dos etapas: modulación y potencia. La simulación del sistema completo se resume en el diagrama de la Fig. 6.

\section{A. Etapa de modulación}

Los algoritmos de las estrategias de modulación para el rectificador y los inversores se programan como funciones en el lenguaje propio de programación de Matlab. Este lenguaje de programación es similar al lenguaje $\mathrm{C}$ pero tiene la ventaja que no requiere inicialización de librerías ni de variables locales; tampoco necesita ser compilado. Los algoritmos de modulación programados calculan los ciclos de trabajo para cada pierna del rectificador e inversores del convertidor matricial, y genera las correspondientes señales de disparo de los dispositivos de potencia (IGBT's). Los algoritmos de modulación son ejecutados en Simulink por bloques específicos que permiten llamar funciones de Matlab (bloque Interpreted Matlab Function). Las señales de disparo de los IGBT's, generados por las funciones de modulación, son enviadas a PSim a través de SimCoupler, un módulo que enlaza Simulink y PSim. 


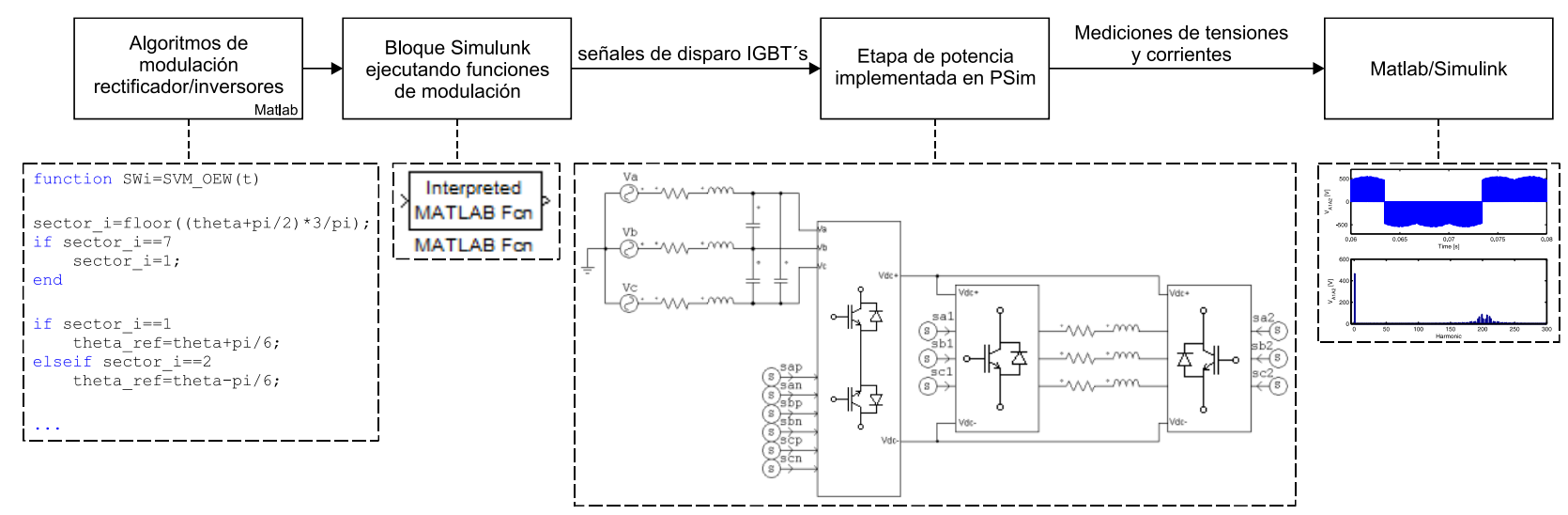

Figura 6. Diagrama de flujo de la simulación realizada.

\section{B. Etapa de potencia}

La etapa de potencia del sistema (fuente de tensión, filtro de entrada, carga y convertidor de potencia) se implementa en PSim. Este modelo recibe las señales de disparo para el IMC a través de In Link Node, un bloque en PSim que provee la interfaz con Simulink. Este bloque recibe un valor desde Simulink y se comporta como una fuente de tensión en PSim. Las mediciones de corrientes y voltajes deseados se realizan en PSim y luego son enviadas a Simulink a través de Out Link Node, otro bloque de PSim usado para transferir valores de variables hacia Simulink. Estos valores pueden ser luego graficados y/o analizados en Matlab.

\section{Resultados de Simulación}

Las estrategias de modulación propuestas para el convertidor se han simulado usando el modelo descrito anteriormente. Por simplicidad la máquina de inducción se modela como una carga R-L trifásica de terminales abiertos. La Tabla III muestra los parámetros de simulación. El índice de modulación usado para la etapa inversora es el máximo posible sin sobremodular. En esta condición, la tensión de salida obtenida en cada fase de la carga para ambas estrategias de modulación es de 1,5 veces la tensión de fase de entrada $(220 \mathrm{~V} \mathrm{rms})$.

\begin{tabular}{cll}
\multicolumn{3}{c}{ TABla III. PARÁMETROS DE Simulación. } \\
\hline Variable & \multicolumn{1}{c}{ Descripción } & Valor \\
\hline$V_{S}$ & Tensión de la fuente & $380 \mathrm{~V}$ \\
$f$ & Frecuencia de la fuente & $50 \mathrm{~Hz}$ \\
$C$ & Capacitancia filtro de entrada & $30 \mu \mathrm{F}$ \\
$L$ & Inductancia filtro de entrada & $0,1 \mathrm{mH}$ \\
$R_{L}$ & Resistencia de carga & $22.6 \Omega$ \\
$L_{L}$ & Inductancia de carga & $72 \mathrm{mH}$ \\
$f_{o}$ & Frecuencia de salida & $50 \mathrm{~Hz}$ \\
$f_{S}$ & Frecuencia de conmutación & $10 \mathrm{kHz}$ \\
\hline
\end{tabular}

Las Figs. 7 y 8 muestran la tensión de línea de salida del inversor $1\left(V_{A 1 B 1}\right)$ para SVM y SPWM. Estas tensiones tienen un valor fundamental de $286 \mathrm{~V}$ ( $\sim 0,75$ veces la tensión de línea de entrada de $380 \mathrm{~V}$ ); sus espectros de frecuencia se muestran en las figuras correspondientes.
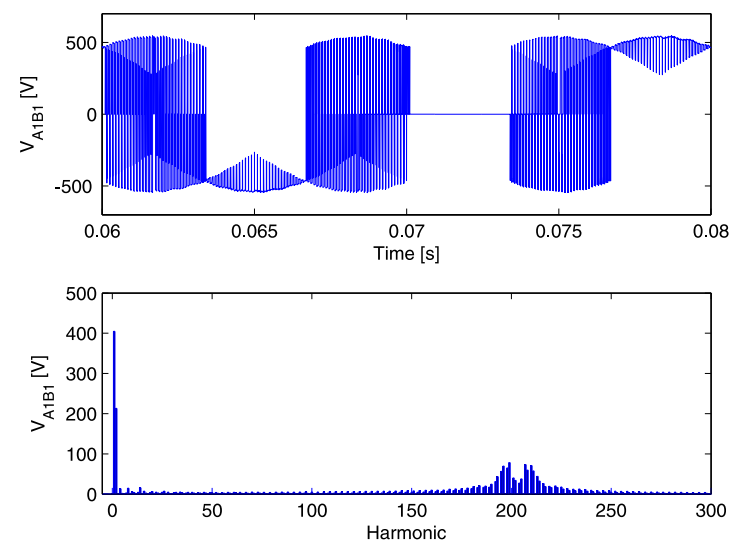

Figura 7. Tensión de línea de salida para SVM.
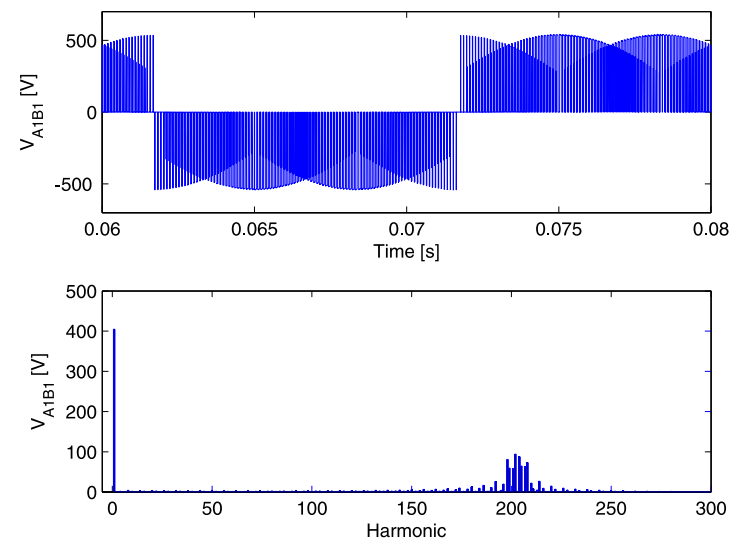

Figura 8. Tensión de línea de salida para SPWM.

La Fig. 9 muestra la tensión en una fase de la carga y su contenido armónico para SVM. La correspondiente tensión para SPWM se muestra en Fig. 10. Esta tensión de fase tiene una componente fundamental de $330 \mathrm{~V}$ ( 1,5 veces la tensión de fase de entrada). La corriente de carga en la fase $a$, similar para ambas estrategas de modulación se muestra en Fig. 11. 


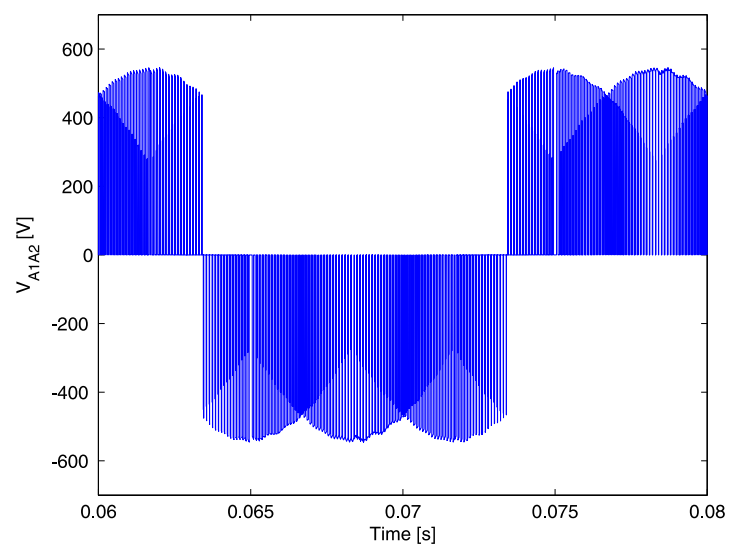

Figura 9. Tensión de fase de salida para SVM.

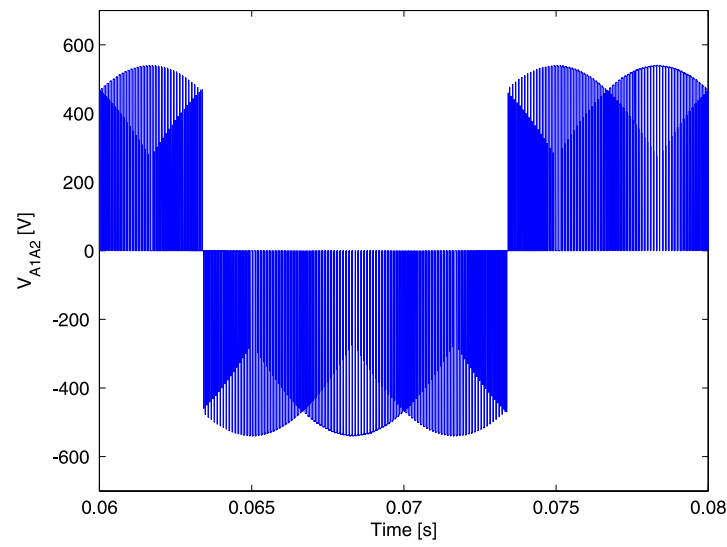

Figura 10. Tensión de fase de salida para SPWM.

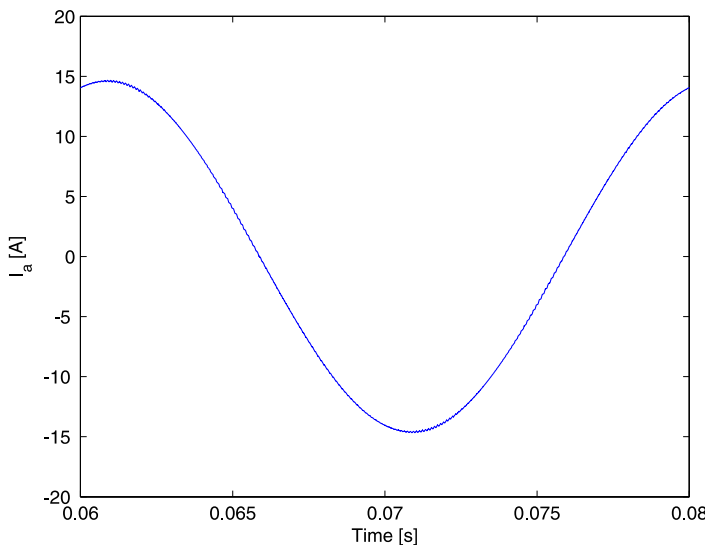

Figura 11. Corriente en la carga para SVM y SPWM.

Las Figs. 12 y 13 muestran la tensión del enlace DC para SVM y SPWM, respectivamente. Puede observarse que para SPWM la tensión DC posee menos rizado comparado con el caso SVM.

La corriente del enlace DC para SVM se muestran en la Fig. 14 junto a su espectro de frecuencia. La misma corriente para el caso SPWM se muestra en la Fig. 15. El espectro armónico de la corriente DC muestra una menor distorsión para el caso SPWM. Esta característica se verá aún más reflejada en la corriente de entrada del convertidor.

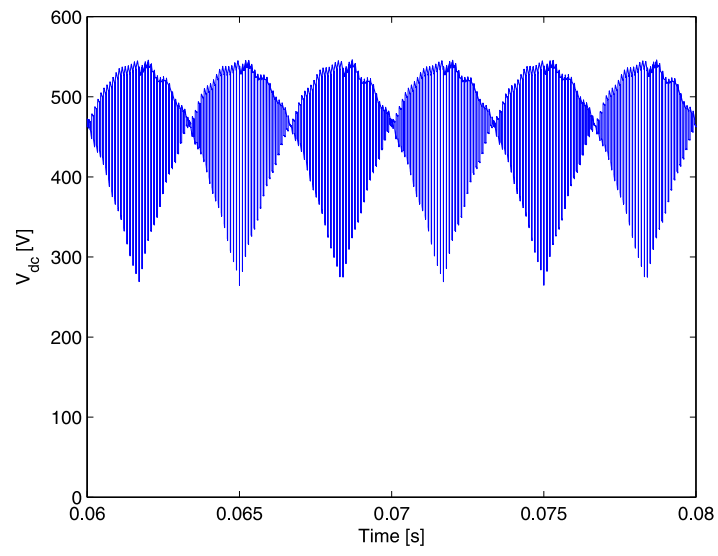

Figura 12. Tensión enlace DC para SVM.

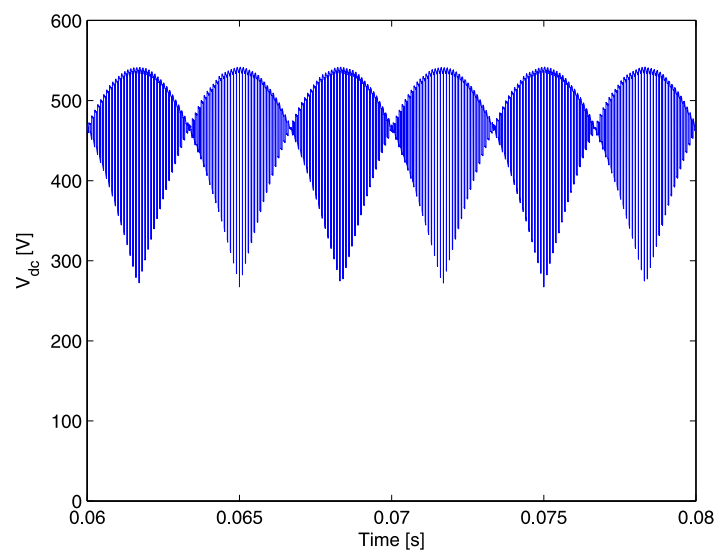

Figura 13. Tensión enlace DC para SPWM.
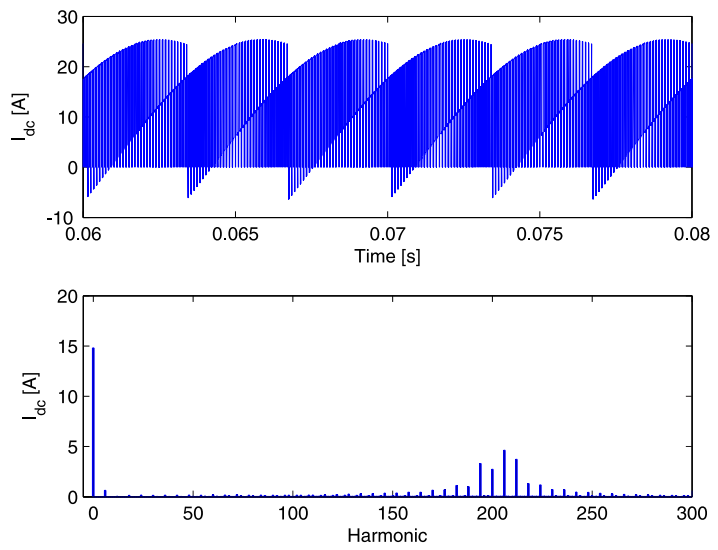

Figura 14. Corriente enlace DC para SVM.

La tensión y corriente de entrada del rectificador para SVM y SPWM se muestran en las Figs. 16 y 17, respectivamente. Puede notarse que el convertidor opera con factor de desplazamiento unitario en la entrada debido a la modulación usada para el rectificador.

La Fig. 18 muestra la corriente consumida de la red para el caso SVM. La misma variable para SPWM se muestra en la Fig. 19. En ambos casos la componente fundamental del espectro de frecuencia ha sido eliminada para mejorar la visualización de los armónicos de bajo orden. Se observa que la estrategia SPWM impone una corriente de entrada menos distorsionada que la estrategia SVM. 

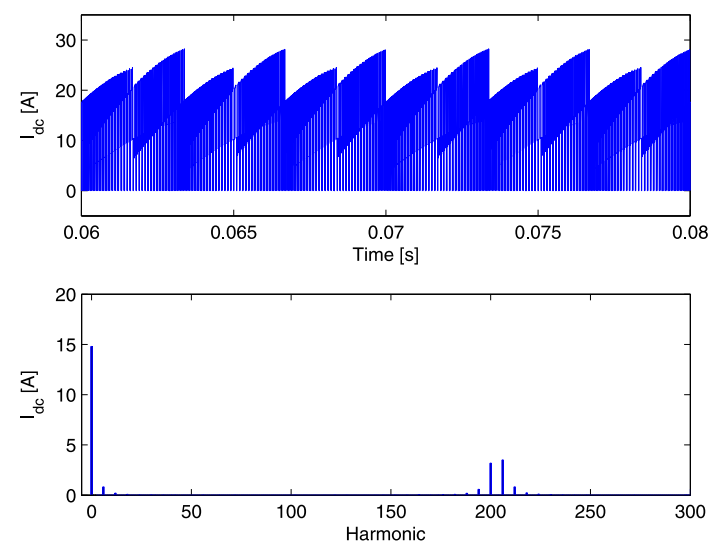

Figura 15. Corriente enlace DC para SPWM.

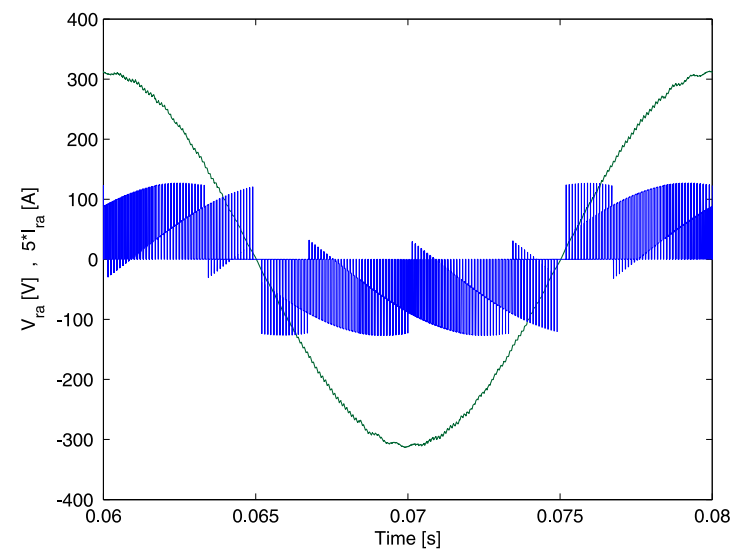

Figura 16. Tensión y corriente de entrada para SVM.

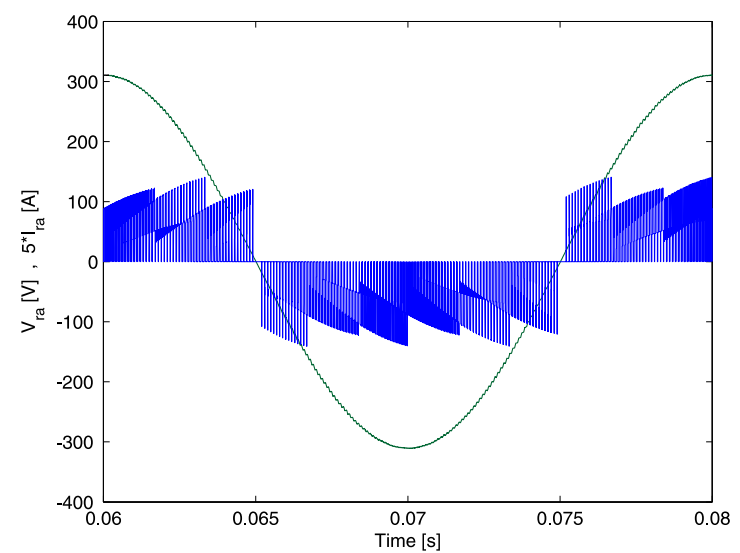

Figura 17. Tensión y corriente de entrada para SPWM.
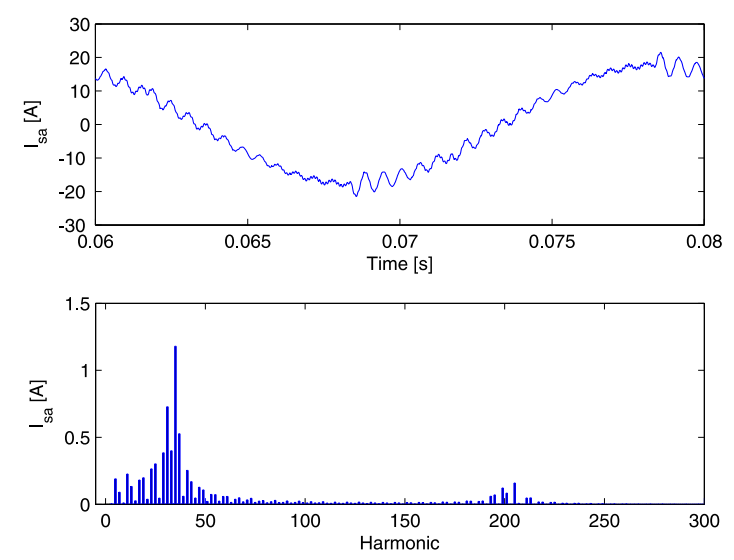

Figura 18. Corriente de la fuente para SVM.
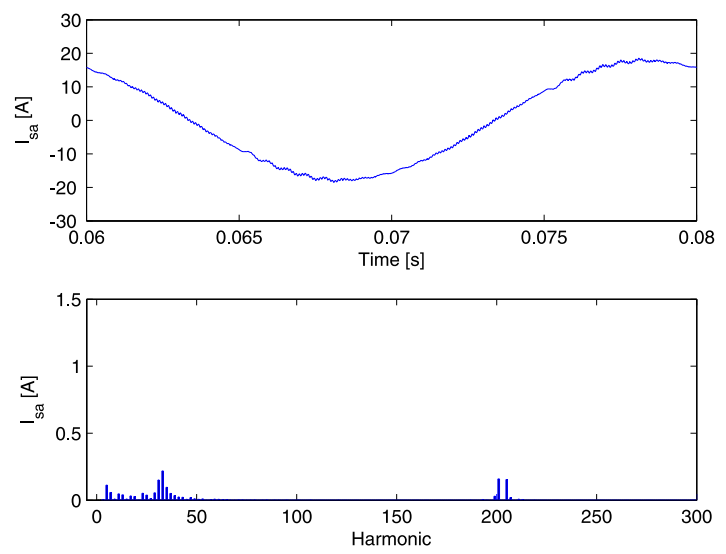

Figura 19. Corriente de la fuente para SPWM.

Finalmente, la tensión de secuencia cero para SVM y SPWM se muestran en las Figs. 20 y 21, respectivamente. Estas tensiones han sido obtenidas midiendo el voltaje en cada fase de la carga y luego aplicando (3). La estrategia SVM no genera tensión de secuencia cero en la carga debido a la selección de vectores de tensión para cada etapa de salida. En la Fig. 21 se observa que la tensión de secuencia cero para SPWM contiene solo componentes de alta frecuencia y debido a la naturaleza inductiva de la carga utilizada, no tiene un efecto apreciable sobre la corriente de salida.

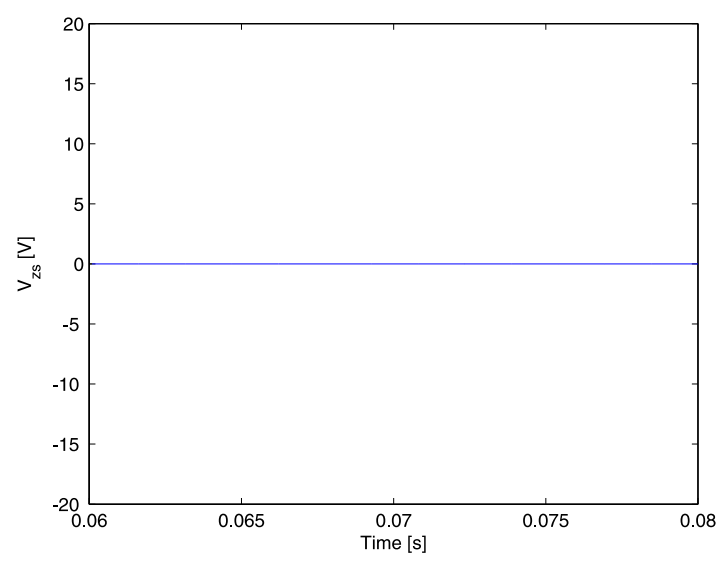

Figura 20. Tensión de secuencia cero para SVM.
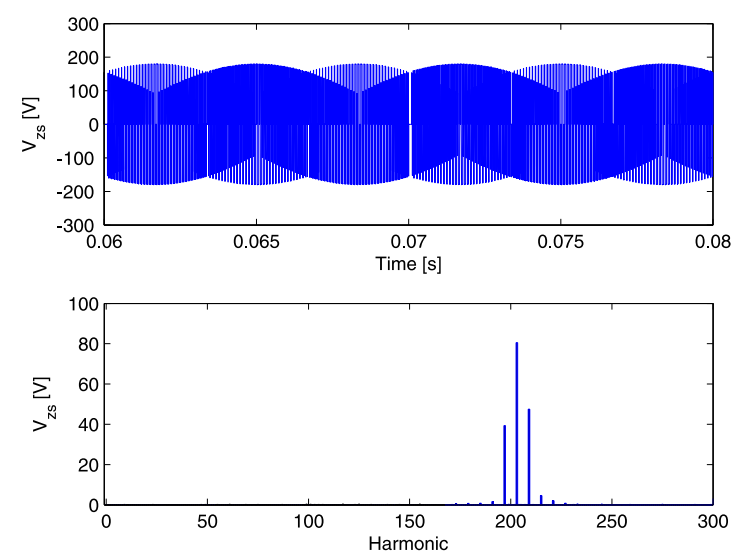

Figura 21. Tensión de secuencia cero para SPWM.

La Tabla IV resume los resultados obtenidos para ambas estrategias de modulación. 
TABLA IV. RESUMEN SIMULACIÓN.

\begin{tabular}{clcc}
\hline \multirow{2}{*}{ Var. } & \multicolumn{1}{c}{ Descripción } & \multicolumn{2}{c}{ Valor RMS } \\
& & SVM & SPWM \\
\hline $\boldsymbol{V}_{\boldsymbol{A 1 \boldsymbol { B } \mathbf { 1 }}}$ & Tensión fund. de línea salida & 286,0 & $286 \mathrm{~V}$ \\
$\boldsymbol{V}_{\boldsymbol{A 1 \boldsymbol { A } \mathbf { 2 }}}$ & Tensión fund. de fase salida & 330,0 & $330 \mathrm{~V}$ \\
$\boldsymbol{I}_{\boldsymbol{a}}$ & Corriente de salida & 10,3 & $10,3 \mathrm{~V}$ \\
$\boldsymbol{I}_{\boldsymbol{a} \boldsymbol{a}}$ & Fund. corriente de entrada & 11,0 & $11,0 \mathrm{~A}$ \\
$\boldsymbol{I}_{\boldsymbol{s} \boldsymbol{a}}$ & Fund. corriente de la fuente & 12,6 & $12,6 \mathrm{~A}$ \\
$\boldsymbol{T} \boldsymbol{H} \boldsymbol{D}_{\boldsymbol{I}_{\boldsymbol{s}}}$ & Distorsión corriente fuente & $9,88 \%$ & $2,28 \%$ \\
\hline
\end{tabular}

\section{CONCLUSIÓN}

Se han presentado dos estrategias de modulación para un Convertidor Matricial Indirecto con dos etapas de salida. La topología tiene como objetivo alimentar máquinas de inducción de terminales abiertos. En las simulaciones mostradas se ha modelado la máquina como carga R-L. Una de las estrategias de modulación se basa en vectores espaciales (SVM) y elimina la tensión de secuencia cero. La otra estrategia mostrada se basa en portadora triangular (SPWM) y genera tensión de secuencia cero de alta frecuencia, produciendo un efecto despreciable en la corriente de salida debido a la naturaleza inductiva de la carga. La estrategia SPWM resulta en una corriente de entrada menos distorsionada que el caso SVM. Ambas estrategias logran una tensión de fase de salida de hasta 1,5 veces la tensión de fase de entrada. La co-simulación Matlab/Simulink-PSim ha mostrado ser una poderosa herramienta para el desarrollo de estrategias de control en circuitos electrónicos de potencia.

\section{AGRADECIMIENTOS}

Este trabajo fue financiado por Fondecyt Chile bajo el contrato 1121104. También se agradece el aporte financiero otorgado por CONICYT/FONDAP/15110019.

\section{REFERENCIAS}

[1] Senicar, F.; Junge, C.; Gruber, S.; Soter, S.; "Zero sequence current elimination for dual-inverter fed machines with open-end windings," IECON 2010 - 36th Annual Conference on IEEE Industrial Electronics Society, vol., no., pp.853-856, 7-10 Nov. 2010.

[2] Somani, A.; Gupta, R.K.; Mohapatra, K.K.; Basu, K.; Mohan, N.; "Modulation strategies for direct-link drive for open-end winding AC machines," Electric Machines and Drives Conference, 2009. IEMDC '09. IEEE International , vol., no., pp.1863-1868, 3-6 May 2009.

[3] P. Wheeler, J. Rodriguez, J. Clare, L. Empringham, and A. Weinstein, "Matrix converters: a technology review," IEEE Transactions on industrial electronics, vol. 49, no. 2, pp. 276-288, 2002.

[4] Klumpner, C.; Blaabjerg, F.; , "Modulation method for a multiple drive system based on a two-stage direct power conversion topology with reduced input current ripple," Power Electronics, IEEE Transactions on , vol.20, no.4, pp. 922- 929, July 2005.

[5] Wei, L.; Lipo, T.A.; , "A novel matrix converter topology with simple commutation," Industry Applications Conference, 2001. Thirty-Sixth IAS Annual Meeting. Conference Record of the 2001 IEEE , vol.3, no., pp.1749-1754 vol.3, Sept. 30 2001-Oct. 42001.

[6] Somasekhar, V.T.; Srinivas, S.; Prakash Reddy, B.; Nagarjuna Reddy, C.; Sivakumar, K.; "Pulse width-modulated switching strategy for the dynamic balancing of zero-sequence current for a dual-inverter fed openend winding induction motor drive," Electric Power Applications, IET , vol.1, no.4, pp.591-600, July 2007.

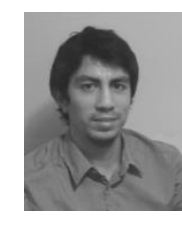

Javier Riedemann Aros nació el 15 de Abril de 1986 en Punta Arenas, Chile. Recibió el título de Ingeniero Eléctrico de la Universidad de Magallanes en Punta Arenas, Chile, en 2010 Actualmente es académico del Departamento de Ingeniería Eléctrica y Electrónica de la Universidad del Bío-Bío, Concepción, Chile, y estudiante del programa de Doctorado en Ingeniería Eléctrica de la Universidad de Concepción, Concepción, Chile. Sus áreas de interés incluyen control de convertidores de potencia y accionamientos de velocidad variable.

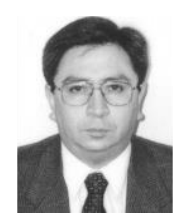

Rubén Peña Guíñez nació el 19 de Agosto de 1960 en Coronel, Chile. Recibió el título de Ingeniero Eléctrico de la Universidad de Concepción en Concepción, Chile, en 1984, y los grados M.Sc. y Ph.D. de la Universidad de Nottingham, Nottingham, Reino Unido, en 1992 y 1996, respectivamente. De 1985 a 2008 se desempeña como académico en la Universidad de Magallanes, Punta Arenas, Chile. Actualmente se desempeña como Profesor Asociado del Departamento de Ingeniería Eléctrica de la Universidad de Concepción. Sus intereses principales son el control de convertidores de potencia, accionamientos de corriente alterna y sistemas de energía renovable.

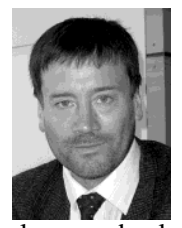

Roberto Cárdenas Dobson nació el 9 de Septiembre de 1961 en Punta Arenas, Chile. Recibió el título de Ingeniero Eléctrico de la Universidad de Magallanes, Punta Arenas, Chile en 1988 y los grados M.Sc. y Ph.D. de la Universidad de Nottingham, Nottingham, Reino Unido, en 1992 y 1996, respectivamente. Entre 1989-1991 y 1996-2008 se desempeña como académico en la Universidad de Magallanes. De 1991 a 1996 perteneció el grupo de electrónica de potencia, máquinas y control de la Universidad de Nottingham. Actualmente es profesor asociado del Departamento de Ingeniería Eléctrica de la Universidad de Chile, Santiago, Chile. Sus intereses incluyen control de máquinas eléctricas, accionamientos de velocidad variable y sistemas de energía renovable. El Dr. Cárdenas recibió el premio "Best Paper Award" de IEEE TRANSACTIONS ON INDUSTRIAL ELECTRONICS en 2004 y el premio "Ramón Salas Edwards" por excelencia en investigación del Instituto de Ingenieros de Chile en 2009.

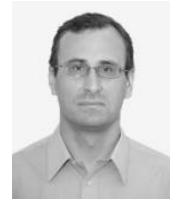

Ramón Blasco-Giménez recibió el grado B.Eng. en ingeniería eléctrica de la Universidad Politécnica de Valencia Valencia, España en 1992 y el grado Ph.D. en ingeniería eléctrica y electrónica de la Universidad de Nottingham, Nottingham, Reino Unido, en 1996. De 1992 a 1995 fue asistente de investigación en el Departamento de Ingeniería Eléctrica y Electrónica de la Universidad de Nottingham. En 1996 se unió al Departamento de Ingeniería de Sistemas y Automática de la Universidad Politécnica de Valencia donde actualmente es profesor titular. Sus intereses incluyen control de accionamientos eléctricos, generación eólica e integración a la red de sistemas de energía renovable. Dr. Blasco-Giménez recibió el premio "Best Paper Award" de IEEE TRANSACTIONS ON INDUSTRIAL ELECTRONICS en 2005, en calidad de co-autor. Es ingeniero profesional registrado en España y miembro de la Institución de Tecnología e Ingeniería en Reino Unido.

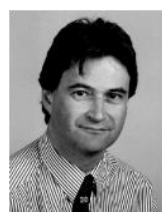

Jon Clare nació en Bristol, Inglaterra. Recibió los grados B.Sc. y Ph.D. en ingeniería eléctrica de la Universidad de Bristol, Reino Unido. De 1984 a 1990 trabajó como académico y asistente de investigación en la Universidad de Bristol en el área de electrónica de potencia. Desde 1990 ha sido parte del grupo de electrónica de potencia, máquinas y control de la Universidad de Nottingham, Nottingham, Reino Unido, y actualmente es profesor en electrónica de potencia. Sus intereses incluyen convertidores electrónicos de potencia y estrategias de modulación, accionamientos de velocidad variable y compatibilidad electromagnética. Dr. Clare es miembro de la Institución de Tecnología e Ingeniería. 